\title{
Histone deacetylation of NIS promoter underlies BRAF V600E-promoted NIS silencing in thyroid cancer
}

\author{
Zongjing Zhang ${ }^{1,2}$, Dingxie Liu', Avaniyapuram Kannan Murugan', Zhimin Liu ${ }^{2}$ and \\ Mingzhao Xing ${ }^{1}$ \\ ${ }^{1}$ Division of Endocrinology, Diabetes, and Metabolism, Laboratory for Cellular and Molecular Thyroid \\ Research, Johns Hopkins University School of Medicine, 1830 East Monument Street, Suite 333, Baltimore, \\ Maryland 21287, USA \\ ${ }^{2}$ Department of Endocrinology and Metabolism, Changzheng Hospital, the Second Military Medical University, \\ Shanghai, China
}

Correspondence should be addressed to $M$ Xing

Email

mxing1@jhmi.edu

\begin{abstract}
The BRAF V600E mutation causes impaired expression of sodium iodide symporter (NIS) and radioiodine refractoriness of thyroid cancer, but the underlying mechanism remains undefined. In this study, we hypothesized that histone deacetylation at the NIS (SLC5A5) promoter was the mechanism. Using the chromatin immunoprecipitation approach, we examined histone acetylation status on the lysine residues $\mathrm{H} 3 \mathrm{~K} 9 / 14, \mathrm{H} 3 \mathrm{~K} 18$, total $\mathrm{H} 4$, and $\mathrm{H} 4 \mathrm{~K} 16$ at the NIS promoter under the influence of BRAF V600E. We found that expression of stably or transiently transfected BRAF V600E inhibited NIS expression while the deacetylase inhibitor SAHA stimulated NIS expression in PCCL3 rat thyroid cells. Although BRAF V600E enhanced global histone acetylation, it caused histone deacetylation at the NIS promoter while SAHA caused acetylation in the cells. In human thyroid cancer BCPAP cells harboring homozygous BRAFV600E mutation, BRAF V600E inhibitor, PLX4032, and MEK inhibitor, AZD6244, increased histone acetylation of the NIS promoter, suggesting that BRAF V600E normally maintained histone in a deacetylated state at the NIS promoter. The regions most commonly affected with deacetylation by BRAF V600E were the transcriptionally active areas upstream of the translation start that contained important transcription factor binding sites, including nucleotides $-297 /-107$ in the rat NIS promoter and $-692 /-370$ in the human NIS promoter. Our findings not only reveal an epigenetic mechanism for BRAF V600E-promoted NIS silencing involving histone deacetylation at critical regulatory regions of the NIS promoter but also provide further support for our previously proposed combination therapy targeting major signaling pathways and histone deacetylase to restore thyroid gene expression for radioiodine treatment of thyroid cancer.
\end{abstract}

\author{
Key Words \\ - thyroid cancer \\ - NIS gene \\ - BRAF V600E mutation \\ - histone acetylation \\ - histone deacetylation \\ - radioiodine
}

Endocrine-Related Cancer (2014) 21, 161-173

\section{Introduction}

$B R A F$ V600E mutation is the most common genetic alteration in thyroid cancer, particularly papillary thyroid cancer (PTC; Xing 2005), and plays an important role in thyroid tumorigenesis through aberrantly activating the RAS-BRAF-MEK-MAP kinase (MAPK) pathway (Xing 2013). Numerous studies around the world have demonstrated http://erc.endocrinology-journals.org DOI: 10.1530/ERC-13-0399
(C) 2014 The authors Published by Bioscientifica Ltd Printed in Great Britain

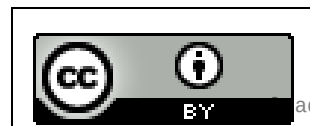

This work is licensed under a Creative Commons Attribution 3.0 Unported License. 
an aggressive role of this mutation in the progression and aggressiveness, including increased recurrence, of PTC (Xing 2007a, Kim et al. 2012, Xing et al. 2013a). A strong association of BRAF V600E mutation with PTC-related mortality has also been recently demonstrated in a large multicenter study (Xing et al. 2013b). Unique molecular derangements caused by BRAF V600E/MAPK pathway are the mechanisms for this aggressive role of BRAF V600E (Xing 2013). Among these is the impairment of the iodidehandling machinery of thyroid cells, as reflected by the initial observation of a strong association of BRAF V600E mutation with the loss of radioiodine avidity in PTC (Xing et al. 2005), a phenomenon that has been confirmed in numerous subsequent studies (Xing 2007a, Kim et al. 2012, Xing et al. 2013a). This phenomenon is clinically relevant, as it suggests an increased risk of radioiodine treatment failure of BRAF V600E-positive PTC, providing an explanation for the association of this mutation with increased disease recurrence and patient mortality of PTC. Correspondingly, numerous studies have reported an association of BRAF V600E with decreased or lost expression of thyroid iodide-handling genes in PTC, particularly sodium iodide symporter (NIS; Xing 2007a, Kim et al. 2012, Xing et al. 2013a), which is normally localized in the basal membrane of thyroid cells and function to transport iodide from the blood stream to the intracellular compartment of the thyroid cell for thyroid hormone synthesis. Our previous studies have shown that introduced expression of BRAF V600E in thyroid cells could induce the silencing of various thyroid iodidehandling genes, most prominently the NIS gene (Liu et al. 2007). BRAF V600E expression could also cause mislocalization of NIS in the cytoplasm in addition to its decreased expression in thyroid cells (Riesco-Eizaguirre et al. 2006). In in vitro cell line assays, inhibition of the BRAF V600E/MEK pathway or silencing of BRAF V600E expression could restore the expression of thyroid genes, particularly NIS in thyroid cells (Liu et al. 2007), which provided important therapeutic implications for targeting the BRAF V600E/MAPK pathway to restore thyroid gene expression and radioiodine avidity of radioiodinerefractory thyroid cancer. This in vitro finding of the reversibility of BRAF V600E-induced silencing of thyroid genes was recently reproduced in an in vivo transgenic mouse model (Chakravarty et al. 2011). However, even given all these exciting achievements, a critical question remains open as to what the molecular mechanism underlies the downregulation of thyroid genes by the BRAF V600E/MAPK pathway.
Histone acetylation is an important epigenetic event that plays a fundamental role in the regulation of gene expression (Li et al. 2007, Dhall \& Chatterjee 2011, Woo \& Li 2012, Horikoshi 2013). It typically occurs at multiple lysine residues in the N-terminal domain of $\mathrm{H} 3$ (Lys 4, 9, 14, 18, 23, and 27) and H4 (Lys 5, 8, 12, and 16) (Kuo et al. 1996, Dhall \& Chatterjee 2011). Histone acetylation and deacetylation are associated with gene transcriptional activation and repression, respectively, representing a fundamental mechanism in aberrant gene activities that promote human tumorigenesis (Barneda-Zahonero \& Parra 2012, Horikoshi 2013). This has been particularly well established for the acetylation sites H3K9/14, H3K18, and $\mathrm{H} 4 \mathrm{~K} 16$ of histones.

Histone acetylation and deacetylation are reversible and are catalyzed by histone acetyltransferases and histone deacetylases (HDACs) respectively. It has been recently hypothesized that BRAF V600E/MAPK pathway might downregulate histone acetylation as a mechanism involving aberrant silencing of thyroid iodide-handling genes in thyroid cancer (Xing 2013). In fact, we demonstrated that the inhibition of HDAC could synergize the inhibitors of BRAF V600E/MAPK pathways to robustly increase thyroid gene expression and radioiodine uptake in thyroid cancer cells (Hou et al. 2010) and even in certain nonthyroid epithelial cancer cells (Hou et al. 2009, Liu \& Xing 2012). This result not only had strong implications for the therapeutic use of HDAC inhibitors to restore radioiodine avidity in thyroid cancer, but was also consistent with the hypothesis of impairment of histone acetylation as a mechanism in BRAF V600E/MAPK pathway-induced thyroid gene silencing. Puzzlingly, however, one study demonstrated that BRAF V600E was in fact associated with increased global histone acetylation in thyroid tumor tissues and in thyroid cell lines expressing BRAF V600E (Puppin et al. 2011). This finding was apparently inconsistent with the above hypothesis. To solve this puzzle, in this study, we tested the possibility that BRAF V600E/MAPK pathway might specifically downregulate histone acetylation at critical regulatory regions of the gene promoter. To test this possibility, we have particularly focused on the NIS gene in this study as it is the most important gene for thyroid uptake of iodide.

\section{Materials and methods}

\section{Cell culture and reagents}

Rat thyroid PCCL3 cell line and its transfectants were cultured and maintained at $37^{\circ} \mathrm{C}$ in $\mathrm{H} 4$ complete medium

Published by Bioscientifica Ltd. 
of Coon's modification of Ham's F-12 (F6636, Sigma) supplemented with $5 \% \mathrm{CO}_{2}, 5 \%$ fetal bovine serum, $1 \mathrm{mIU} / \mathrm{ml} \mathrm{TSH}, 5 \mu \mathrm{g} / \mathrm{ml}$ apo-transferrin, $10 \mu \mathrm{g} / \mathrm{ml}$ insulin, and $10 \mathrm{nM}$ hydrocortisone. PCCL3/BRAF cells conditionally expressed $\mathrm{BRAF}^{\mathrm{V} 600 \mathrm{E}}$ or WT-BRAF induced by $1 \mu \mathrm{g} / \mathrm{ml}$ doxycycline (DOX; Sigma) for $48 \mathrm{~h}$. The HDAC inhibitor SAHA (Sigma) at $0.5 \mu \mathrm{M}$ was used to treat cells in some experiments. DMSO or PBS was used as a vehicle control.

Noninducible parental rat PCCL3 cells were transiently transected with various BRAF protein-expressing plasmid constructs using the Lipofectamine 2000 transfection reagent following the manufacturer's instructions (Invitrogen, Life Technologies) and the cells were harvested $48 \mathrm{~h}$ after transfection.

The human thyroid cancer cell line, BCPAP, was cultured in RPMI 1640 supplemented with 2 mM L-glutamine, 10\% fetal bovine serum, MEM nonessential amino acids (\#25-005$\mathrm{Cl}, 100 \times)$, and $1 \mathrm{mM}$ sodium pyruvate (\#25-000-Cl) (all from Coring, Cellgro, Manassas, VA, USA). AZD6244 (Selleck Chemicals, Houston, TX, USA) and PLX4032 (Plexxikon, Inc., Berkeley, CA, USA) were dissolved in DMSO and used to treat cells at $1 \mu \mathrm{M}$ in some experiments.

\section{Western blotting}

The cells were lysed in RIPA lysis buffer (RIPA) (sc-24948, Santa Cruz Biotechnology) containing standard protease inhibitor cocktails. After measurement and adjustment of protein concentration using the Bio-Rad Dc Protein Assay, $15 \mu \mathrm{g}$ of each sample was separated on 10\% SDS-PAGE, transferred onto PVDF membrane (Amersham Pharmacia Biotech), and blocked with 5\% blotting milk (170-6404, Blotting Grade Blocker, Bio-Rad) for $1 \mathrm{~h}$. The following primary antibodies were used for immunoblotting: anti-cMyc (9E10) (sc-40, Santa Cruz Biotechnology), anti-Raf-B (C-19) (sc-166, Santa Cruz Biotechnology), anti-p-ERK (E-4) (sc-7383, Santa Cruz Biotechnology), anti- $\beta$ actin (I-19) (sc-1616, Santa Cruz Biotechnology), and anti-H3K9/14 (06599B, Millipore, Billerica, MA, USA). The cells were incubated overnight with primary antibodies at $4{ }^{\circ} \mathrm{C}$. After washing with TBST three times, each for $5 \mathrm{~min}$, the blots were incubated with HRP-linked anti-mouse or anti-rabbit second antibody for $2 \mathrm{~h}$ at room temperature, followed by three 5-min washings. Protein bands in the membrane were visualized with ECL reaction reagents (Amersham Pharmacia Biotech).

\section{Total RNA isolation, RT-PCR, and quantitative PCR}

Total cellular RNA was isolated in $500 \mu \mathrm{l}$ of Trizol reagent (15596-018, Invitrogen) following the manufactures' instructions. Two microgram of total RNA was reversetranscribed to DNA using the Script cDNA Synthesis Kit (1708891, Bio-Rad Laboratories). Real-time PCR was performed using SYBR Green Supermix (Bio-Rad Laboratories) with $50 \times$ ROX (54881, Invitrogen) on the ABI 7900HT PCR system (Applied Biosystems). $\beta$-actin was run in parallel for quality control. The RT-PCR primers and procedures for rat NIS and $\beta$-actin genes were as described previously (Liu et al. 2007).

\section{Chromatin immunoprecipitation assay}

Chromatin immunoprecipitation (ChIP) assay was performed using the Magna ChIP A kit (17-408, Millipore) according to the manufacturers' protocol as described previously (Liu \& Xing 2012). Briefly, protein-DNA was cross-linked by incubating cells with diluted formaldehyde for $10 \mathrm{~min}$. Glycine was then added for 5 min to quench unreacted formaldehyde. After washing with PBS twice, cells were lysed and sonicated ten times each for $10 \mathrm{~s}$ with $20 \mathrm{~s}$ rest between pulses at a $25 \%$ pulse power using the Branson Sonifier (150D Liquid Processor). Chromatin was sheared to lengths between 200 and $1000 \mathrm{bp}$, mostly between 200 and $500 \mathrm{bp}$. Cross-linked protein-DNA complexes were incubated overnight at $4{ }^{\circ} \mathrm{C}$ with anti-histone acetylation antibodies, including antiacetylated H3K9/14 (06-599B, Millipore), anti-acetylated H3K18 (9675s, Cell Signaling), anti-acetylated H4 (17-630, Millipore) (the acetyl-histone $\mathrm{H} 4$ antiserum was made against a peptide corresponding to amino acids 2-19 of tetrahymena histone $\mathrm{H} 4$, which is conserved in eukaryotes and contains the four histone acetylation sites H4K5, H4K8, H4K12, H4K16), anti-acetylated H4K16 (17-10101, Millipore), normal rabbit IgG (PP64B), and fully suspended protein A magnetic beads. After washing protein A beads-antibody/chromatin complexes with $800 \mu \mathrm{l}$ washing buffer, cross-linked protein-DNA complexes were reversed by incubating with proteinase $\mathrm{K}$ at $62{ }^{\circ} \mathrm{C}$ for $3 \mathrm{~h}$ with gentle shaking. DNA was purified using spin columns. Seven pairs of rat NIS primers and five pairs of human NIS primers, corresponding to the defined regions of the NIS promoters (Table 1), were used to perform real-time quantitative PCR (qPCR) to detect DNA fragments obtained from ChIP. Real-time qPCR was carried out with an initial denaturation at $95^{\circ} \mathrm{C}$ for $10 \mathrm{~min}$, followed by 50 cycles of denaturation at $95^{\circ} \mathrm{C}$ for $30 \mathrm{~s}$, and annealing and extension at $60^{\circ} \mathrm{C}$ for $1 \mathrm{~min}$. Data were normalized using the $\beta$-actin gene. The results of ChIP assays were expressed as the fraction of the input DNA. Threshold cycles $(\mathrm{Ct})$ were determined for ChIP samples and the input DNA, and the relative amount of

Published by Bioscientifica Ltd. 
Table 1 qPCR primers of NIS promoters for ChIP studies

\begin{tabular}{|c|c|c|c|}
\hline Type of NIS & Promoter regions & Primer sequence $\left(5^{\prime}-3^{\prime}\right)$ & $\begin{array}{l}\text { Amplicon size }(\mathrm{bp}) \text { and } \\
\text { nucleotide number }\end{array}$ \\
\hline \multirow[t]{7}{*}{ Rat NIS } & Exon 1 & $\begin{array}{l}\text { F: TCACCGAGTCACCTGTCTCCA } \\
\text { R: TGCACAGCCGACATGAAACT }\end{array}$ & $124(-20 / 104)$ \\
\hline & P1 & $\begin{array}{l}\text { F: CACAACCCTATACGGAACAAG } \\
\text { R: TTCGCGCTGCGGATTTATTG }\end{array}$ & $190(-297 /-107)$ \\
\hline & P2 & $\begin{array}{l}\text { F: CAAGCTGCGGAGAAAGGTAGA } \\
\text { R: CACAACCCTATACGGAACAAG }\end{array}$ & $200(-477 /-277)$ \\
\hline & P3 & $\begin{array}{l}\text { F: GACAGCCAGGTCAGGACAACATG } \\
\text { R: GAGCATCTACCTTTCTCCGCAGC }\end{array}$ & $226(-678 /-452)$ \\
\hline & P4 & $\begin{array}{l}\text { F: ATGCAACACCAGCTCCAG } \\
\text { R: GTGGGCTCATGATGTATATG }\end{array}$ & $174(-1124 /-950)$ \\
\hline & P5 & $\begin{array}{l}\text { F: ACCAAGCTTTAGGTGAGGACT } \\
\text { R: TCTGTGGCCAGTAGAGACTTGA }\end{array}$ & $161(-1874 /-1713)$ \\
\hline & NUE & $\begin{array}{l}\text { F: GAATCAGGAGGTTCTACAGT } \\
\text { R: CTTGATCTTGGAGTCCTGT }\end{array}$ & $285(-2627 /-2342)$ \\
\hline \multirow[t]{5}{*}{ Human NIS } & Exon 1 & $\begin{array}{l}\text { F: CTGGGACTACGGGGTCTTTGC } \\
\text { R: TGCCAGTGGGGCAGGTCCTA }\end{array}$ & $237(42 / 399)$ \\
\hline & P1 & $\begin{array}{l}\text { F: GAGTGCTGAAGCAGGCTGTGC } \\
\text { R: GGGAGCAGCTCGTGATTGTGG }\end{array}$ & $323(-692 /-370)$ \\
\hline & P2 & $\begin{array}{l}\text { F: CTGGCACAGGGCCAACTCTCA } \\
\text { R: TCAGGGTTTCAGGGGACCCATA }\end{array}$ & $385(-1147 /-762)$ \\
\hline & P3 & $\begin{array}{l}\text { F: CTGACGCTGTTTCTTTCACCC } \\
\text { R: GACCACCAGGGAGGTAGAGTC }\end{array}$ & $296(-1511 /-1216)$ \\
\hline & NUE & $\begin{array}{l}\text { F: GAGCCCTCAGGCAGTTGCT } \\
\text { R: ACTCACGTGGAACTGCTTGA }\end{array}$ & $238(-9525 /-9287)$ \\
\hline
\end{tabular}

NIS, sodium iodide symporter; $F$, forward; $R$, reverse. Nucleotide number is defined by having the first nucleotide of the translation initiation codon as +1 (Puppin et al. 2012, Woo \& Li 2012.

immunoprecipitated DNA (\% ChIP signal per input DNA) was calculated as $100^{2 \Delta C t}$ (Puppin et al. 2012).

\section{Gene promoter area prediction}

To identify critical regulatory regions of the NIS promoter for histone acetylation studies, we carried out prediction analyses for the promoter transcriptional activities and response elements of rat and human NIS genes and designed the primers for these regions as presented in Table 1 in the ChIP studies. The following online tools were used for this purpose:

1. For promoter activity: http://tools.igsp.duke.edu/ generegulation/McPromoter/;

2. For transcription factor-binding site: http://www.cbil. upenn.edu/cgi-bin/tess/tess;

3. For CpG island: http://www.uscnorris.com/cpgislands2/cpg.aspx;

4. For TATA box: http://zeus2.itb.cnr.it/ webgene/ wwwHC_tata.html.

\section{Statistical analysis}

The data presented are representatives of at least three similar experiments. The differences between groups of at

http://erc.endocrinology-journals.org DOI: 10.1530/ERC-13-0399
(C) 2014 The authors Printed in Great Britain least three experiments were analyzed by $t$-test. A $P$ value $<0.05$ was considered statistically significant.

\section{Results}

BRAF V600E suppressed NIS gene expression in rat thyroid cells

We previously demonstrated that induced expression of BRAF V600E in PCCL3/BRAF thyroid cells for 6 days or 30 days resulted in dramatic inhibition of the expression of thyroid genes, particularly NIS (Liu et al. 2007). In this study, we reproduced this result by showing that BRAF V600E could decrease NIS expression even after a 48-h DOX-induced expression of BRAF V600E in PCCL3 cells stably transfected with this oncoprotein (Fig. 1A). In contrast, treatment of cells with the HDAC inhibitor, SAHA, could increase the expression of NIS. To further confirm the specific effect of BRAF B600E on NIS expression, we performed transient expression of BRAF proteins in PCCL3 cells. In comparison with the empty vector transfection, transient expression of BRAF V600E for $48 \mathrm{~h}$ significantly decreased NIS expression in PCCL3 cells, whereas expression of the WT-BRAF had no effect on

Published by Bioscientifica Ltd 

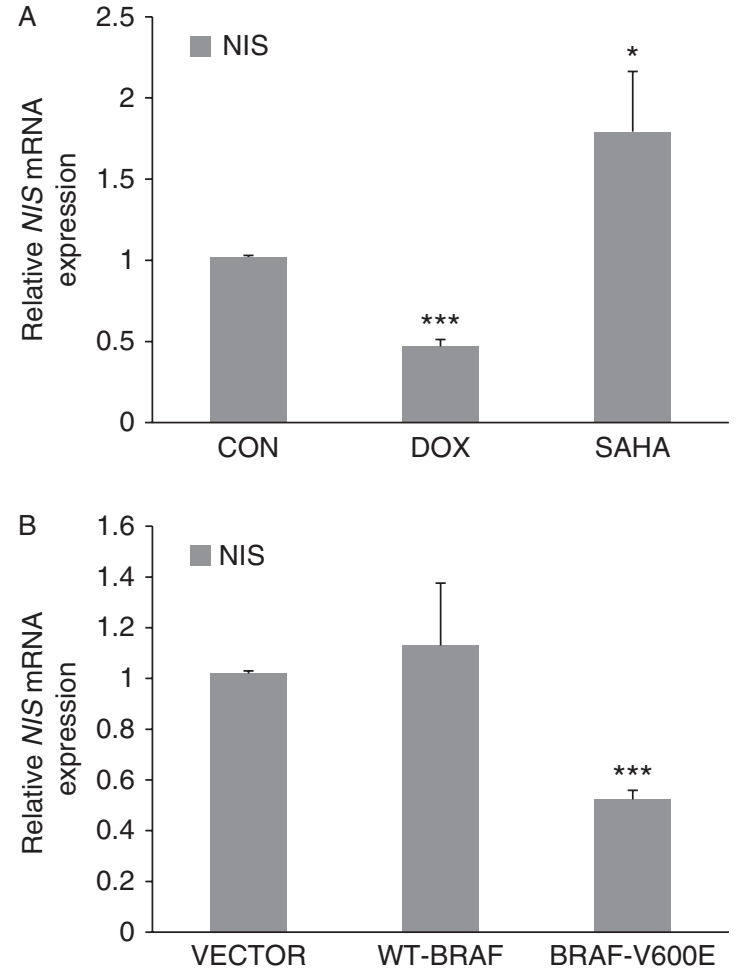

C

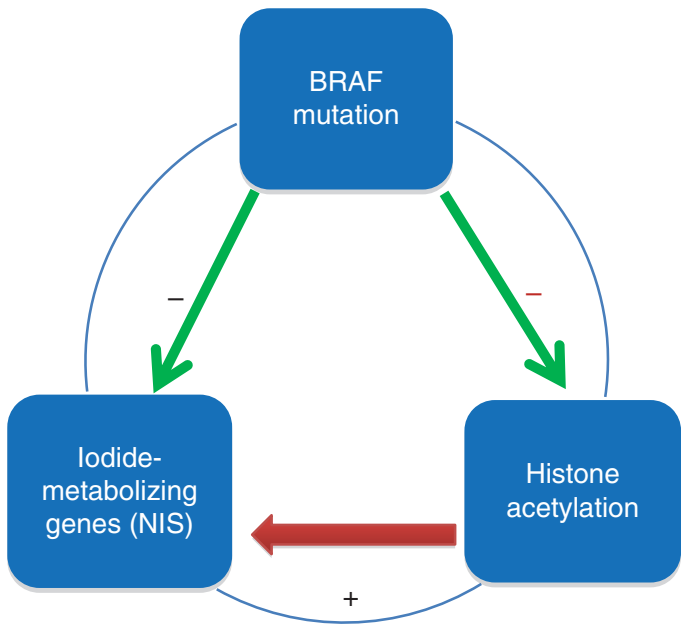

Figure 1

Effect of BRAF V600E on the expression of the NIS gene in rat thyroid cells. (A) In PCCL3/BRAF cells, DOX at $1 \mu \mathrm{g} / \mathrm{ml}$ induced BRAFV600E expression and NIS gene mRNA expression was correspondingly decreased by $54 \%$ in $48 \mathrm{~h}$. The histone deacetylases inhibitor, SAHA, at $0.5 \mu \mathrm{M}$ increased NIS mRNA expression in PCCL3/BRAF cells. (B) PCCL3 cells transiently transfected with BRAF V600E showed a decrease in NIS expression by $48 \%$ in $48 \mathrm{~h}$ compared with the empty vector, and WT-BRAF did not have effect. (C) Hypothesized mechanism for BRAF V600E mutation-induced downregulation of NIS gene expression, in which BRAFV600E downregulated histone acetylation at the NIS promoter and decreased NIS expression. Each bar represents the mean value \pm S.E.M. of three different experiments. CON, control; DOX, doxycycline. ${ }^{*} P<0.05, * * * P<0.001$.
NIS expression (Fig. 1B). The effects of BRAF V600E expression and HDAC inhibition on NIS expression were clearly in opposite directions. Give these results and our previously demonstrated reversibility of BRAF V600E-induced NIS suppression (Liu et al. 2007) as well as the fact that histone acetylation and deacetylation are rapid reversible biochemical modifications, we believed, as recently hypothesized (Xing 2013), that BRAFV600E/MAPK pathway suppressed the expression of NIS by downregulating histone acetylation (Fig. 1C).

\section{BRAF V600E increased global histone acetylation in thyroid cells}

As an initial step to test our hypothesis, we first examined the effect of BRAF V600E on the overall global histone acetylation in thyroid cells. At $48 \mathrm{~h}$ of induction by DOX, abundant expression of stably transfected BRAF V600E was induced, accompanied by a sharp increase in the phosphorylation of ERK (p-ERK; Fig. 2, left panel). Contrary to our expectation based on our hypothesis, the global histone H3K9/14 acetylation of cells was increased following BRAF V600E expression. This was similar to the increased global histone H3K9/14 acetylation induced by treating cells with the HDAC inhibitor, SAHA (Fig. 2, right and left panels). SAHA did not have effect on the expression of BRAF or p-ERK. These results reproduced a similar previous finding of the upregulation of global histone acetylation by stable expression of BRAF V600E in rat thyroid cells (Puppin et al. 2011). Transient expression of BRAF V600E for $48 \mathrm{~h}$ also increased p-ERK accompanied by increased global histone acetylation, while expression of WT-BRAF did not have such effects (Fig. 2, middle panel), confirming the specific effect of BRAF V600E on histone acetylation. Unlike SAHA, DOX itself had no effect on histone acetylation in PCCL3 cells (Fig. 2, right panel), demonstrating that the effect of DOX-induced expression of stably transfected BRAF V600E on histone acetylation represented a real effect of BRAF V600E, but not that of DOX. These results on the effects of BRAF V600E on global histone acetylation did not seem to agree with our hypothesis that BRAF V600E downregulates thyroid genes by causing histone deacetylation. We then hypothesized that perhaps, in contrary to the global histone acetylation, histone acetylation at critical regions of the promoter of NIS gene might be specifically downregulated by BRAF V600E/MAPK pathway, hence

Published by Bioscientifica Ltd. 


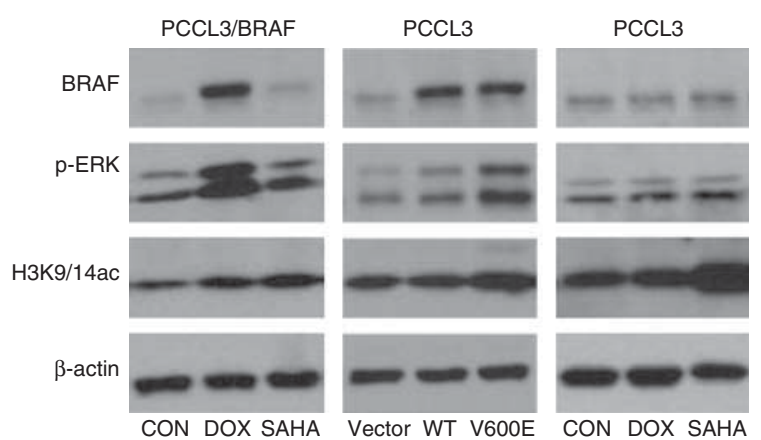

Figure 2

Global histone acetylation changes in PCCL3/BRAF and PCCL3 cells upon various treatments. $10^{6}$ cells were planted in 12 -well plates in each condition. PCCL3/BRAF cells were treated with $1 \mu \mathrm{g} / \mathrm{ml}$ DOX to induce BRAF V600E expression (left), PCCL3 cells were transiently expressed with BRAF V600E (middle), and PCCL3 cells were treated with DOX at $1 \mu \mathrm{g} / \mathrm{ml}$ or SAHA at $0.5 \mu \mathrm{M}$ (right), followed by cell lysis and protein preparation for westernblotting $48 \mathrm{~h}$ later. BRAF V600E expression was successfully induced, accompanied by P-ERK activation and increase in global H3K9/14 acetylation. SAHA at $0.5 \mu \mathrm{M}$ increased global histone H3K9/14 acetylation in both PCCL3/BRAF cells and WT PCCL3 cells. PCCL3 cells treated with DOX at $1 \mu \mathrm{g} / \mathrm{ml}$ did not show change in histone $\mathrm{H} 3 \mathrm{~K} 9 / 14$ acetylation, unlike treatment with SAHA. CON, control; DOX, doxycycline; p-ERK, phosphorylated ERK; H3K9/14ac, acetylated H3K9/14.

negatively affecting the expression of NIS. We hereafter focused on testing this possibility.

\section{Suppression of H3K9/14 acetylation of histone at the NIS promoter by BRAF V600E}

To investigate specifically the acetylation status of histone at NIS promoter, we performed ChIP studies using real-time qPCR primers designed to target several regions of the promoter, including exon 1, P1, P2, P3, P4, and P5 as presented in Table 1. As shown in Fig. 3A, DOX-induced expression of stably transfected BRAF V600E significantly suppressed H3K9/14 acetylation of histone at P1, P2, and P3 in PCCL3 cells. The acetylation was slightly suppressed at exon 1, P4, and P5. No change was seen at the distantly located region of nuclear upstream enhancer (NUE). In contrast and as expected, SAHA increased H3K9/14 acetylation of histone uniformly at all these regions of the NIS promoter. Upon transient transfection of PCCL3 cells for 48 h, BRAF V600E significantly suppressed H3K9/14 acetylation of histone at exon 1 and P1, but the empty plasmid vector or WT-BRAF did not have such an effect (Fig. 3B). In these transient transfection experiments, we did not see significant change in $\mathrm{H} 3 \mathrm{~K} 9 / 14$ acetylation of histone at P2, P3, P4, P5, and NUE in PCCL3 cells. One possible explanation is the less robust expression of BRAF and p-ERK in transient transfection than in stable transition of the cells (Fig. 2).

\section{Suppression of H3K18 acetylation of histone at the NIS promoter by BRAF V600E}

As shown in Fig. 4A, DOX-induced expression of stably transfected BRAF V600E significantly suppressed H3K18 histone acetylation at the $\mathrm{P} 1$ and $\mathrm{P} 2$ regions of the NIS promoter in PCCL3/BRAF cells. In contrary, SAHA significantly increased H3K18 histone acetylation at P1. H3K18 histone acetylation was not affected at other regions of NIS promoter. Transient expression of BRAF V600E significantly suppressed H3K18 histone acetylation at P1 of the NIS promoter (Fig. 4B). In contrast, transient expression of the WT-BRAF or transfection with the empty vector did not affect H3K18 acetylation at P1. H3K18 histone acetylation at other regions of the NIS promoter did not show suppression by BRAF V600E.

\section{Suppression of total $\mathrm{H} 4$ histone acetylation at the NIS promoter by BRAF V600E}

As shown in Fig. 5A, DOX-induced expression of stably transfected BRAF V600E significantly suppressed total H4 acetylation at the $\mathrm{P} 2$ region of the NIS promoter in PCCL3/BRAF cells, whereas SAHA significantly increased total $\mathrm{H} 4$ acetylation at the $\mathrm{P} 2$ region. BRAF V600E also slightly suppressed $\mathrm{H} 4$ acetylation at exon 1 and P1, but this was not statistically significant. SAHA also increased total $\mathrm{H} 4$ histone acetylation at the P1 region. SAHA slightly increased $\mathrm{H} 4$ histone acetylation at the P3 region, but this was not significant. Transient expression of BRAF V600E in PCCL3 cells suppressed total H4 acetylation at P1, whereas transient expression of the WT-BRAF or transfection with the empty vector did not affect $\mathrm{H} 4$ acetylation (Fig. 5B). Other regions of the NIS promoter were not affected by transient expression of BRAF V600E.

\section{Suppression of H4K16 histone acetylation of the NIS promoter by BRAF V600E}

We also examined specifically the acetylation of K16 of H4 at the NIS promoter. As shown in Fig. 6A, DOX-induced expression of stably transfected BRAF V600E significantly suppressed H4K16 acetylation at the P1 and P3 regions of the NIS promoter in PCCL3/BRAF cells, whereas SAHA significantly increased H4K16 acetylation at the two regions. Like the increase in the global histone acetylation by BRAF V600E (Fig. 2), DOX-induced expression of BRAF V600E also increased H4K16 acetylation at the NUE region (Fig. 6A). Transient expression of BRAF V600E in PCCL3 cells suppressed H4K16 acetylation at P1, P3, and P5,

Published by Bioscientifica Ltd. 

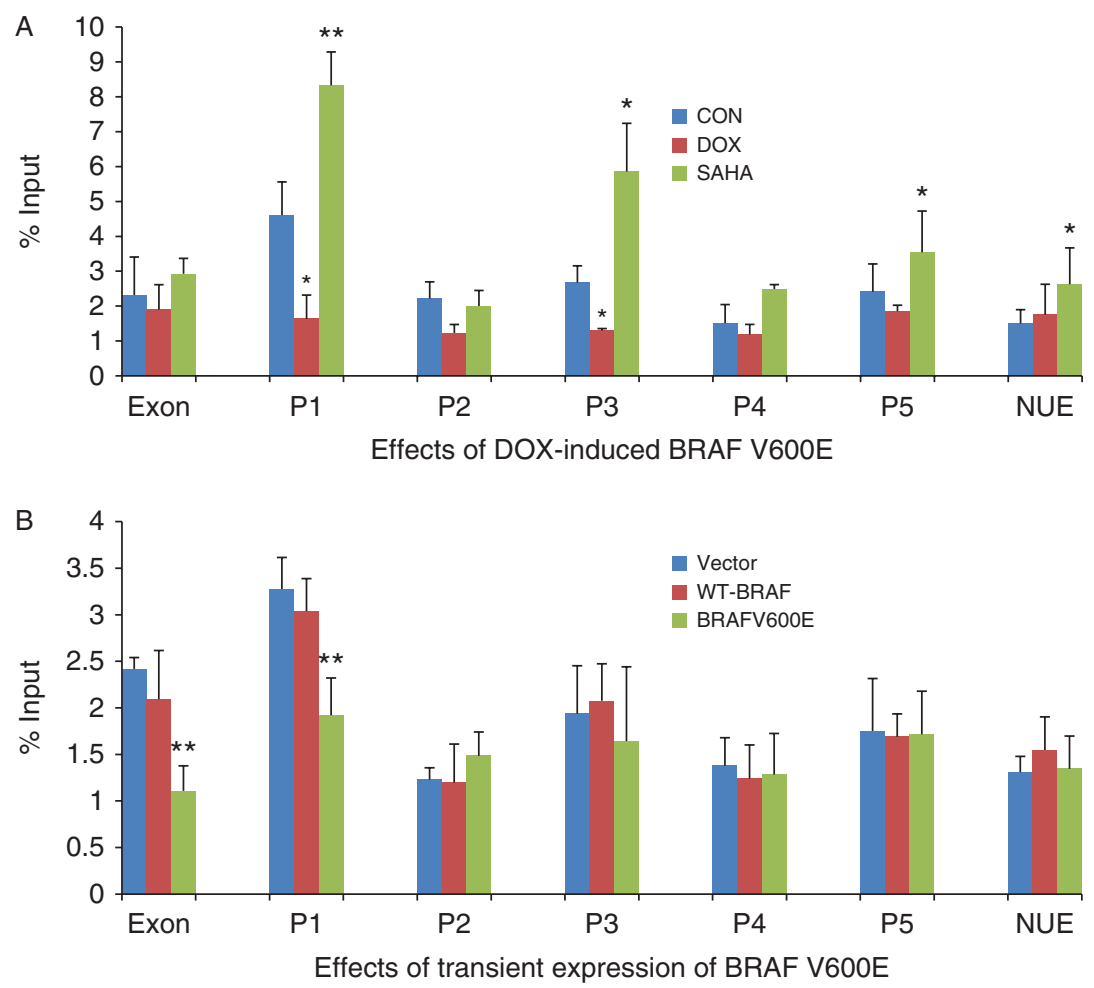

\section{Figure 3}

Effect of BRAF V600E on H3K9/14 acetylation in the rat NIS promoter in rat thyroid cells. (A) In PCCL3/BRAF cells, DOX at $1 \mu \mathrm{g} / \mathrm{ml}$ induced BRAF V600E expression after $48 \mathrm{~h}$, accompanied by decrease in $\mathrm{H} 3 \mathrm{~K} 9 / 14$ acetylation in the regions $\mathrm{P} 1, \mathrm{P} 2$, and $\mathrm{P} 3$ of the NIS promoter, most dramatically in $\mathrm{P} 1$. SAHA at $0.5 \mu \mathrm{M}$ increased $\mathrm{H} 3 \mathrm{~K} 9 / 14$ acetylation in regions $\mathrm{P} 1, \mathrm{P} 3, \mathrm{P} 4$, and NUE. (B) In PCCL3 cells, transient expression of BRAF V600E decreased H3K9/14 acetylation at exon 1 and P1 of the NIS promoter while transfection with WT-BRAF had no effect. Histone acetylation status was analyzed by ChIP. The levels of H3K9K14 acetylation are expressed as fraction of the input DNA (material before immunoprecipitation). Threshold cycles $(\mathrm{Ct})$ were determined for ChIP samples and the input DNA, and the relative amount of immunoprecipitated DNA (\% ChIP signal per input DNA) was calculated as $100^{2 \Delta C t}$. Each bar represents the mean value \pm S.E.M. of at least three different experiments. The promoter regions are as presented in Table 1. EXON, exon 1; NUE, nuclear upstream enhancer; CON, control; DOX, doxycycline; rNIS, rat NIS. ${ }^{*} P<0.05, * * P<0.01$.

whereas transient expression of the WT-BRAF or transfection with the empty vector did not affect histone acetylation at these regions of NIS promoter in PCCL3 cells (Fig. 6B). Other regions of the NIS promoter were not affected by transient expression of BRAF V600E.

\section{The role of BRAF V600E in modulating histone acetylation of human NIS promoter}

The results presented above obtained from rat thyroid cells indicated an important role of the BRAF V600E/MEK pathway in the modulation of histone acetylation of NIS promoter. To confirm that this is the case in human thyroid cancer cells, we used human thyroid cancer cell line BCPAP to test the role of BRAF/MAPK pathway in modulating histone acetylation of human NIS promoter. BCPAP cells harbor homozygous BRAF V600E mutation (Liu \& Xing 2008). As shown in Fig. 7A, the BRAF/MEK

http://erc.endocrinology-journals.org DOI: 10.1530/ERC-13-0399
(C) 2014 The authors Printed in Great Britain pathway was inhibited, as reflected by the suppression of p-ERK, by treatment of BCPAP cells with the MEK inhibitor, AZD6244 (AZD), or the BRAF V600E inhibitor, PLX4032 (PLX). The overall histone H3K9/14 acetylation was not affected by these treatments. SAHA did not affect p-ERK, but it significantly increased the overall acetylation of histone H3K9/14. To test the role of BRAF/MAPK pathway in modulating histone acetylation specifically at the human NIS promoter, we similarly treated BCPAP cells with AZD and PLX and selectively examined the acetylation status of two acetylation sites of histone, H3K9/14 and H4K16, at the NIS promoter. As for the rat NIS promoter, for the human NIS promoter we also examined several critical regions, including exon 1, P1, P2, P3, and NUE as presented in Table 1. As shown in Fig. 7B, treatment of cells with AZD resulted in a significant increase in H3K9/14 acetylation at regions P1 and P2. SAHA similarly increased H3K9/14 acetylation at

Published by Bioscientifica Ltd. 

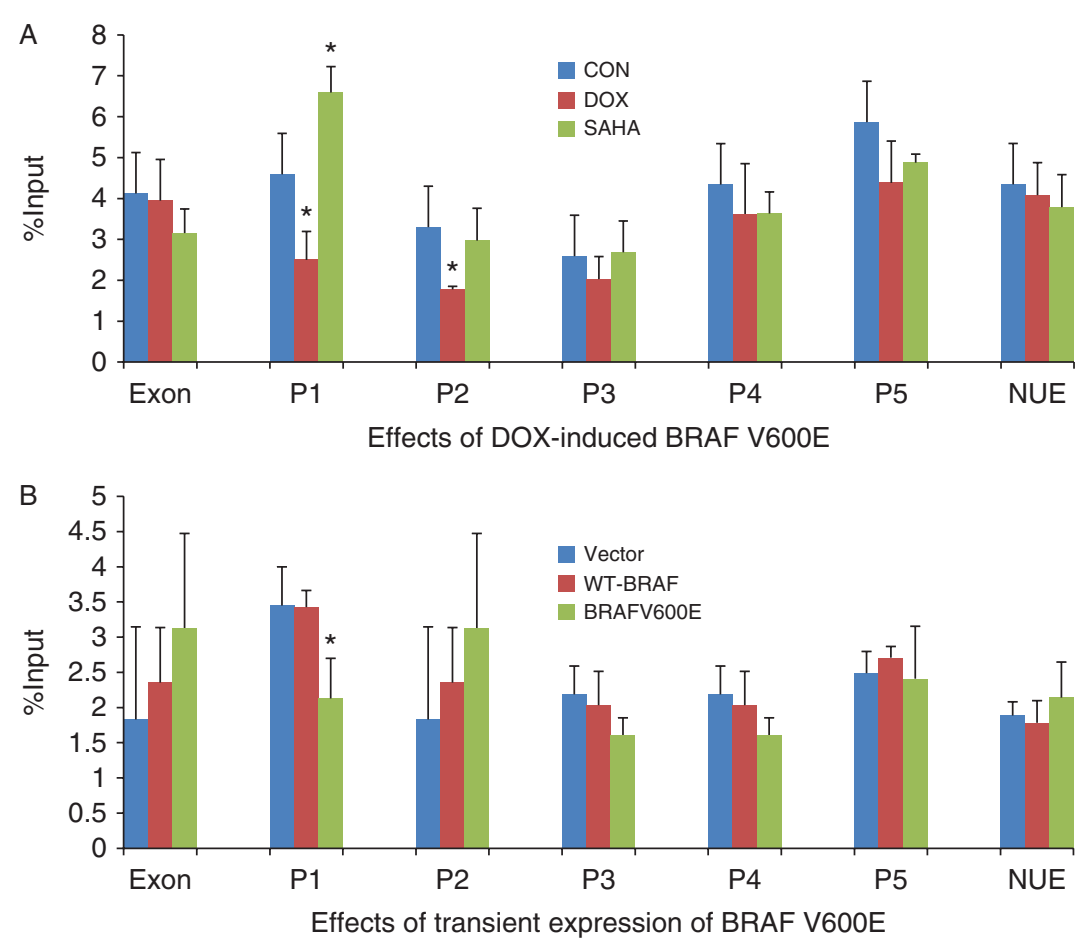

\section{Figure 4}

Effect of BRAF V600E on H3K18 acetylation in the rat NIS promoter in rat thyroid cells. (A) In PCCL3/BRAF cells, DOX at $1 \mu \mathrm{g} / \mathrm{ml}$ induced BRAF V600E expression after $48 \mathrm{~h}$, accompanied by decrease in H3K18 acetylation in regions $\mathrm{P} 1$ and $\mathrm{P} 2$ of the NIS promoter. SAHA at $0.5 \mu \mathrm{M}$ increased H3K18 acetylation in regions P1. (B) In PCCL3 cells, transient expression of BRAF V600E decreased H3K18 acetylation at P1 of the NIS promoter while

the two regions. Treatment of cells with PLX significantly increased H3K9/14 acetylation at P1 (Fig. 7B). Histone acetylation at other regions of the NIS promoter in BCPAP cells was not affected by these treatments. Similarly, AZD and PLX both caused a significant increase in the acetylation of $\mathrm{H} 4 \mathrm{~K} 16$ at region P1 (Fig. 7C). In contrast, acetylation of H4K16 at other regions was not affected. SAHA increased the acetylation of H4K16 at all the five regions of the human NIS promoter. These results suggest that the BRAF V600E/MAPK pathway normally inhibits histone acetylation at the human NIS promoter in thyroid cancer cells and removal of this inhibition increased histone acetylation at the NIS promoter.

\section{Discussion}

In this present study, we for the first time demonstrated downregulation of histone acetylation specifically at the promoter of both the rat and human NIS genes by BRAF V600E. Histone acetylation at the promoter area is a wellestablished mechanism in the upregulation of genes, transfection with WT-BRAF had no effect. Results of ChIP assays were expressed as the fraction of the input DNA. Each bar represents the mean value \pm s.E.M. of at least three different experiments. The promoter regions are as presented in Table 1. Definitions of other symbols are as in Fig. 3. ${ }^{*} P<0.05$.

which, through chromatin remodeling, opens up the access of gene promoters to transcription factors (Li et al. 2007, Dhall \& Chatterjee 2011, Horikoshi 2013). Conversely, histone deacetylation causes compacting of chromatin and blocking of gene promoters from binding with transcription factors, resulting in gene silencing. There are multiple lysine residues in the N-terminal tail of histone that are the sites of acetylation, including H3K9, H3K14, H3K18, and H4K16. This study showed that histone deacetylation occurred on all these lysine residues of histones at the NIS promoter upon the activation of MAPK by BRAF V600E. Both previous (Puppin et al. 2011) and this studies demonstrated an upregulation of global histone acetylation by BRAF V600E, which initially seemed to be puzzlingly against our proposed mechanism in which BRAF V600Emediated downregulation of NIS gene involves deacetylation of histone in thyroid cancer. This puzzle was solved when we looked at the histone acetylation specifically at the promoter of the NIS gene and found histone deacetylation at critical regulatory regions of the NIS

Published by Bioscientifica Ltd. 

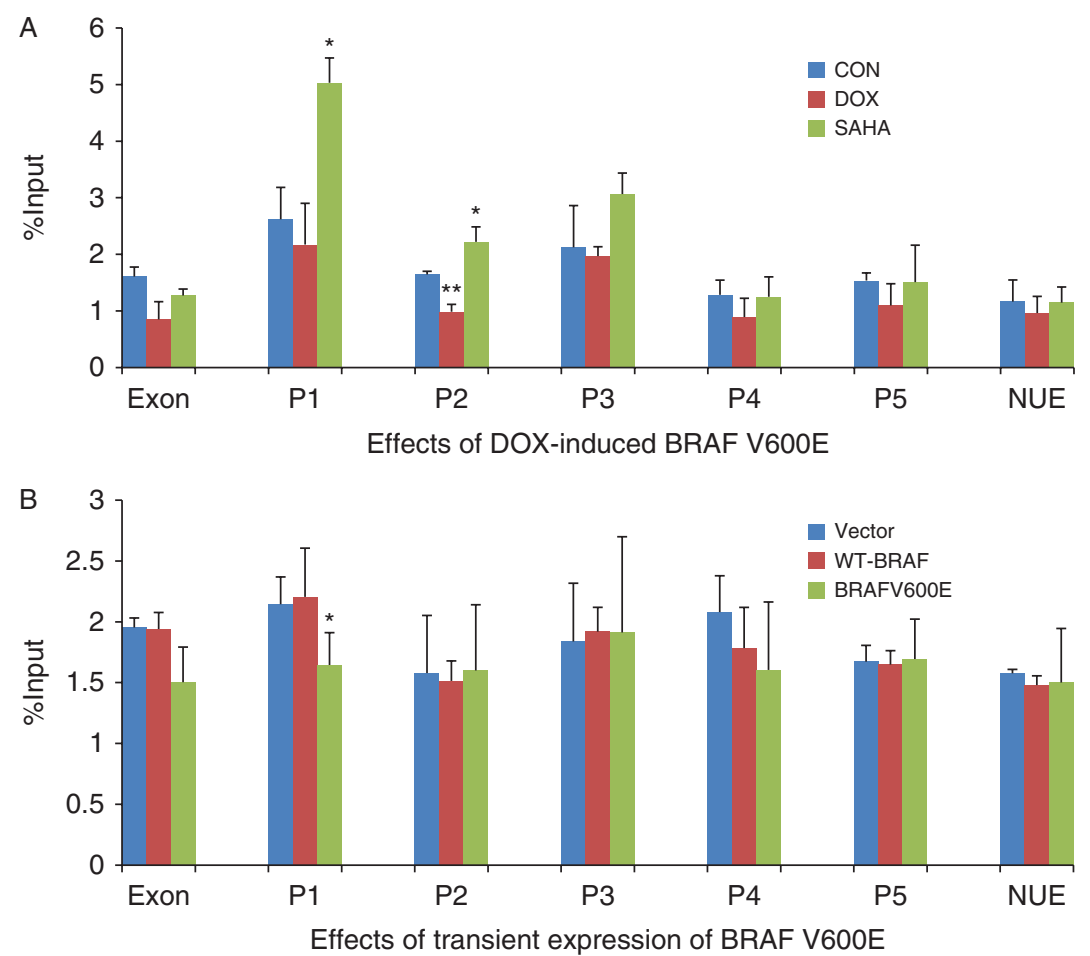

\section{Figure 5}

Effect of BRAF V600E on $\mathrm{H} 4$ acetylation in the rat NIS promoter in rat thyroid cells. (A) In PCCL3/BRAF cells, DOX-induced BRAF V600E expression caused a decrease in $\mathrm{H} 4$ acetylation at $\mathrm{P} 2$ of the rat NIS promoter and SAHA at $0.5 \mu \mathrm{M}$ increased $\mathrm{H} 4$ acetylation at $\mathrm{P} 1$ and $\mathrm{P} 2$ of the rat NIS promoter. (B) In PCCL3 cells, transient expression of BRAF V600E decreased H4

gene. This discordant relations between global histone acetylation and histone deacetylation at a specific gene promoter promoted by an oncogene are similar to another epigenetic phenomenon in human cancer driven by oncogenes, in which global DNA hypomethylation is coupled with hypermethylation of the promoter of tumor suppressor genes (Momparler \& Bovenzi 2000), the latter being associated with gene silencing as an important epigenetic mechanism in thyroid tumorigenesis (Xing 2007b). Upregulation of NIS expression by enhanced histone acetylation has been widely demonstrated in thyroid cells (Puppin et al. 2005, Kogai et al. 2006, Xu \& Hershman 2006, Hou et al. 2010, Pugliese et al. 2013). Thus, the finding of histone deacetylation of NIS promoter by BRAF V600E in this study provides an important epigenetic mechanism for the downregulation of NIS gene in BRAF V600Eharboring thyroid cancer.

Histone modifications are conserved in various species including yeast, mouse, rat, and human and their stability is determined mainly by cell types in which the specific acetylation at P1 of the NIS promoter while transfection with WT-BRAF had no effect. The levels of $\mathrm{H} 4$ acetylation were expressed as fraction of the input DNA. Each bar represents the mean value \pm s.E.M. of 3-5 different experiments. The promoter regions are as presented in Table 1. Definitions of other symbols are as in Fig. $3 .{ }^{\star} P<0.05, * \star P<0.01$.

regulatory mechanisms control histone modifications (Woo \& Li 2012). Therefore, in this study we used rat thyroid cells as a model to study the impact of BRAF V600E/MAPK on histone acetylation of NIS and found similar NIS promoter deacetylation patterns in rat thyroid cells and human thyroid cancer cells. It has long been known that acetylation of both $\mathrm{H} 3$ and $\mathrm{H} 4$ tends to occur at promoters areas of individual genes and such histone acetylation is particularly important compared with global histone acetylation in the regulation of individual genes (Vogelauer et al. 2000). This is consistent with our present finding on the importance of histone acetylation status of the NIS promoter. It is interesting and important to note that among the several regions of NIS promoter, P1, P2, and $\mathrm{P} 3$ were the most commonly affected with histone deacetylation by BRAF V600E. These regions, particularly PI, of NIS promoter are close to the translation initiation site. It is worth noting that histone deacetylation at P1 of the NIS promoter occurred virtually with all the histone lysine residues (H3K9, H3K14, H3K18, and H4K16) examined in rat thyroid cells and human thyroid cancer

Published by Bioscientifica Ltd. 

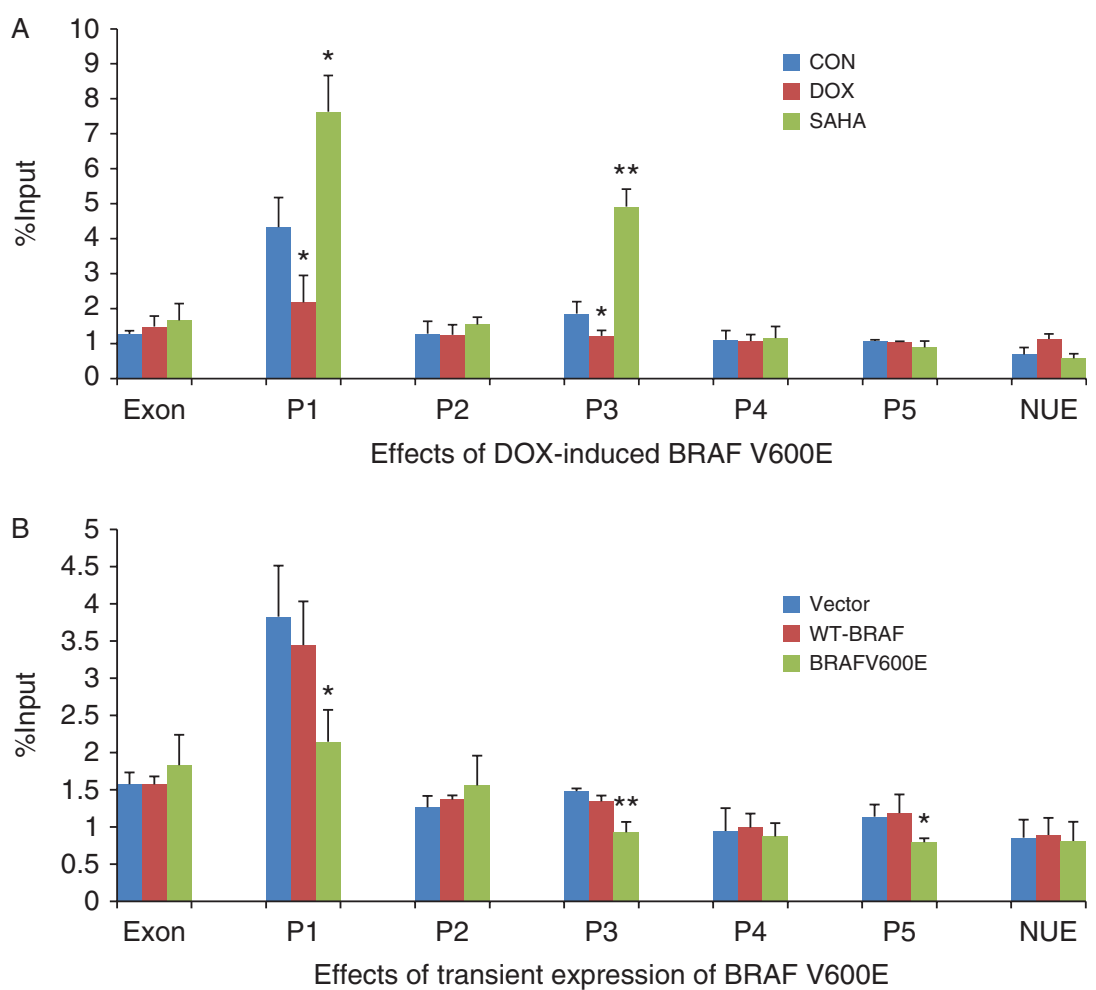

\section{Figure 6}

Effect of BRAF V600E on H4K16 acetylation in the rat NIS promoter in rat thyroid cells. (A) In PCCL3/BRAF cells, DOX-induced BRAF V600E expression caused a decrease in $\mathrm{H} 4 \mathrm{~K} 16$ acetylation at $\mathrm{P} 1$ and $\mathrm{P} 3$ of the rat NIS promoter and increased H4K16 acetylation at NUE of the NIS promoter. SAHA at $0.5 \mu \mathrm{M}$ increased $\mathrm{H} 4 \mathrm{~K} 16$ acetylation at $\mathrm{P} 1$ and $\mathrm{P} 3$ of the rat NIS promoter. (B) In PCCL3 cells, transient expression of BRAF V600E decreased H4K16

cells. These are among the most important lysine residues for the acetylation of histone affecting gene expression in human cancer (Bjerling et al. 2002, Liang et al. 2004, Fraga et al. 2005). These results suggest that histone acetylation/ deacetylation at P1 is particularly important in the regulation of NIS expression and thus histone deacetylation at this critical region of NIS promoter by BRAF V600E could profoundly affect the expression of NIS.

Several important transcriptional factor binding sites have been defined in the NIS promoter, including TATA box, AP1, AP2, GR, TTF1, T3Ra, T3R-b, and SP1 binding sites in rat NIS promoter (Tong et al. 1997) and TATA box, TTF1,AP1, AP2, Sp1, and cAMP response element-binding protein (CREB) binding sites in human NIS promoter (Ryu et al. 1998). The regions P1 $(-297 /-107)$, P2 $(-477 /-277)$, and P3 $(-678 /-452)$ harbor many of these transcription factor-binding sites and are located in the most active region of the rat NIS promoter upstream the translation start site. In rat thyroid cells, DNA fragments $(-490$ to -118$)$ appears to have the strongest acetylation at $\mathrm{P} 1, \mathrm{P} 3$, and $\mathrm{P} 5$ of the NIS promoter while transfection with WT-BRAF had no effect. The levels of H4K16 acetylation were expressed as fraction of the input DNA. Each bar represents the mean value \pm s.E.M. of 3-5 different experiments. The promoter regions are as presented in Table 1. Definitions of other symbols are as in Fig. $3 . * P<0.05, * * P<0.01$.

transcriptional activity and a potential TATA box (AATAAAT) is located at -124 to -118 and a TTF1 binding site is located near -480 (Tong et al. 1997). Thus, regions $\mathrm{P} 1$ and $\mathrm{P} 2$ of NIS promoter play a critical role in the regulation of NIS gene, consistent with the present finding that BRAF V600E/MAPK pathway causes histone deacetylation most profoundly in these regions in rat thyroid cells. In human thyroid cancer cells, treatment with BRAF V600E and MEK inhibitors increased H3K9/14 and H4K16 acetylation mainly at regions P1 $(-692 /-370)$ and P2 $(-1147 /-762)$ of the human NIS promoter, suggesting that BRAF V600E/MEK normally exerts a negative effect on histone acetylation at regions P1 and P2 of the NIS promoter in human thyroid cancer cells. A region $(-475 /-393)$ within P1 of the human NIS promoter has a strong similarity in DNA sequence (72\%) with a region $(-196 /-114)$ within P1 of the rat NIS promoter (Kogai et al. 2006). The DNA sequence of a region $(-790 /-728)$ partially involving P2 of the human NIS promoter has $63.5 \%$ similarity with that of a region $(-422 /-361)$

Published by Bioscientifica Ltd 
A
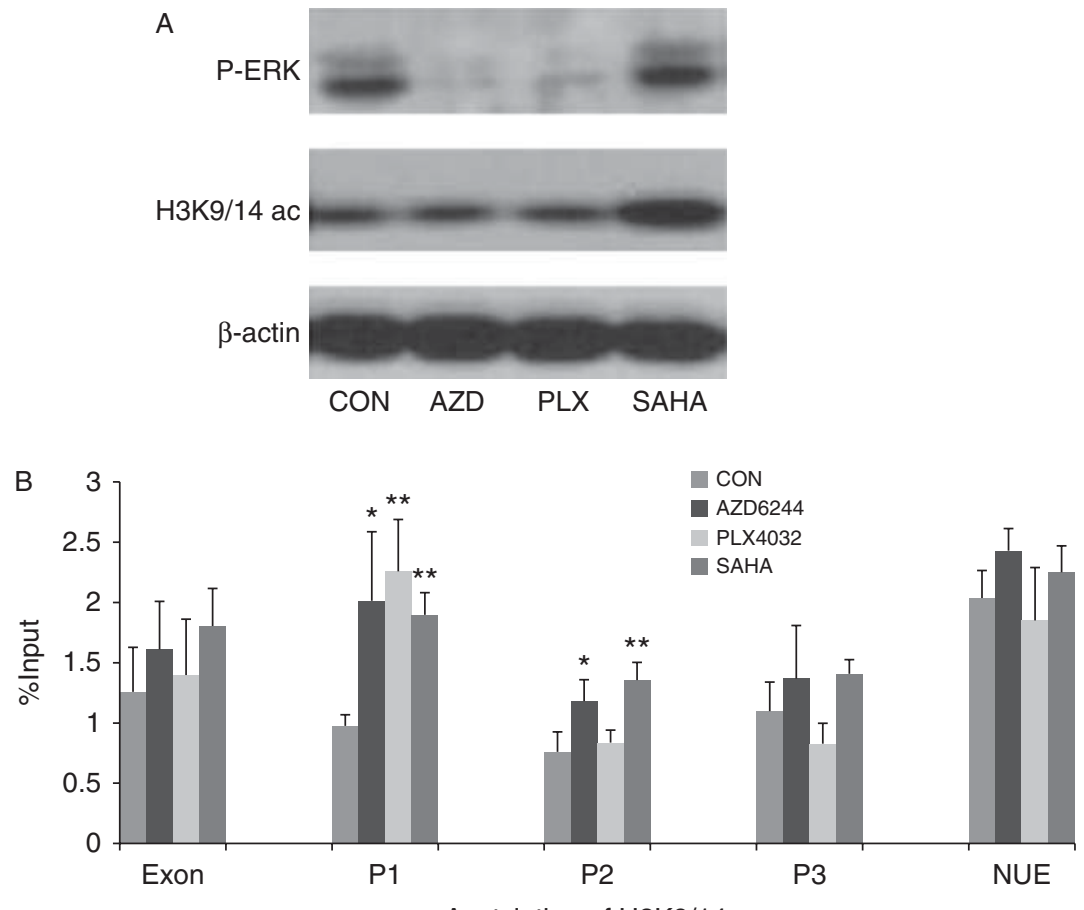

Acetylation of $\mathrm{H} 3 \mathrm{~K} 9 / 14$

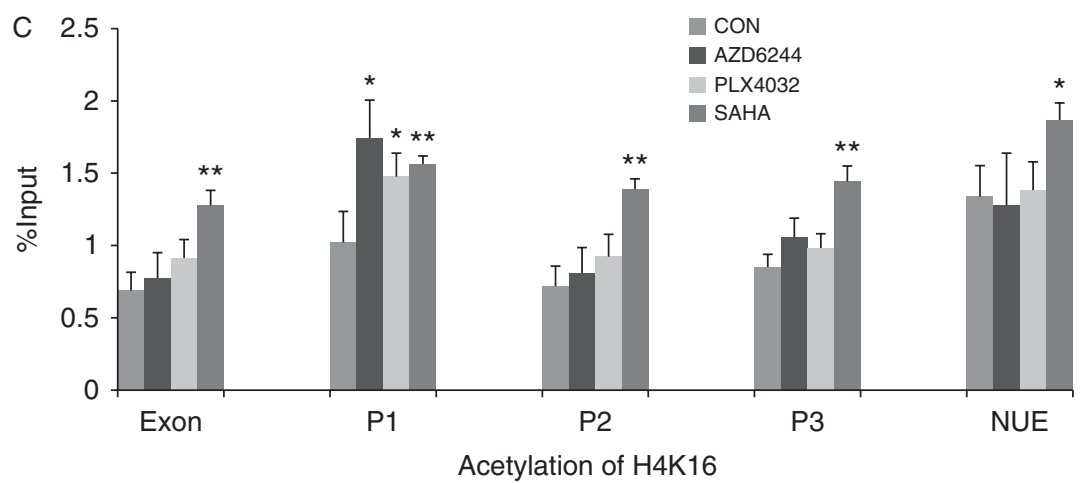

\section{Figure 7}

The role of BRAF V600E/MAP kinase pathway in modulating histone acetylation at the human NIS promoter in thyroid cancer BCPAP cells. (A) Treatment of BCPAP cells with the MEK inhibitor AZD6244 (AZD) at $1 \mu \mathrm{M}$ and the BRAF V600E inhibitor PLX4032 (PLX) at $1 \mu \mathrm{M}$ for $48 \mathrm{~h}$ completely suppressed downstream P-ERK and had no significant effect on global H3K9/14 acetylation. The histone deacetylases inhibitor SAHA at $0.5 \mu \mathrm{M}$ increased global H3K9/14 acetylation in BCPAP cells. (B) Effects of various inhibitors on $\mathrm{H} 3 \mathrm{~K} 9 / 14$ acetylation at the human NIS promoter. ChIP assay was used to analyze histone acetylation. AZD increased H3K9/14 acetylation at regions $\mathrm{P} 1$ and $\mathrm{P} 2, \mathrm{PLX}$ increased $\mathrm{H} 3 \mathrm{~K} 9 / 14$ acetylation at region $\mathrm{P} 1$, and SAHA increased $\mathrm{H} 3 \mathrm{~K} 9 / 14$ acetylation at regions $\mathrm{P} 1$ and $\mathrm{P} 2$ of

within P2 of the rat NIS promoter (Kogai et al. 2006). These are consistent with the similar impact of BRAF V600E on the histone acetylation of NIS promoter and expression of the NIS gene in both rat thyroid cells and human thyroid cancer cells, in this study. the human NIS promoter. (C) Effects of various inhibitors on H4K16 acetylation at the human NIS promoter. Both AZD and PLX increased H4K16 acetylation at region P1 of the human NIS promoter and SAHA increased H4K16 acetylation in all the regions. The levels of histone acetylation were expressed as fraction of the input DNA. Each bar represents the mean value \pm s.E.M. of at least three different experiments. The human NIS promoter regions are as presented in Table 1. P-ERK, phosphorylated ERK; H3K9/14ac, acetylated H3K9/14; CON, control; AZD, AZD6244; PLX, PLX4032. * $P<0.05$, $* * P<0.01$. Full colour version of this figure available via http://dx.doi.org/ 10.1530/ERC-13-0399.

BRAF V600E-promoted loss of expression of NIS and other thyroid genes and hence the development of radioiodine refractoriness currently represent a major therapeutic obstacle for thyroid cancer patients. This study not only uncovers an epigenetic mechanism involving histone

Published by Bioscientifica Ltd 
deacetylation at critical regulatory regions of the NIS promoter for BRAF V600E-promoted NIS silencing but also provides further evidence supporting our previous proposal to use a combination strategy targeting major signaling pathways and HDAC to restore thyroid gene expression and radioiodine avidity (Hou et al. 2010). In this context, the MEK inhibitor AZD, BRAF V600E inhibitor PLX (vemurafenib), and HDAC inhibitor SAHA (vorinostat), which have been approved for the treatment of other human cancers, could reasonably be used as a combination therapy to restore radioiodine avidity for radioiodine treatment of radioiodine-refractory thyroid cancer.

\section{Declaration of interest}

The authors declare that there is no conflict of interest that could be perceived as prejudicing the impartiality of the research reported.

\section{Funding}

This study was supported by grants NIH RO1 CA113507 to M Xing.

\section{Author contribution statement}

$M X$ conceived and designed the experiments. ZZ, DL \& AKM performed the experiments. $Z Z \& M X$ analyzed the data. $M X$ contributed reagents/ materials/analysis tools. ZZ, ZL \& MX wrote the paper.

\section{Acknowledgements}

The authors thank Drs Massimo Santoro and James A Fagin for kindly providing the thyroid cell lines used in this study.

\section{References}

Barneda-Zahonero B \& Parra M 2012 Histone deacetylases and cancer. Molecular Oncology 6 579-589. (doi:10.1016/j.molonc.2012.07.003)

Bjerling P, Silverstein RA, Thon G, Caudy A, Grewal S \& Ekwall K 2002 Functional divergence between histone deacetylases in fission yeast by distinct cellular localization and in vivo specificity. Molecular and Cellular Biology 22 2170-2181. (doi:10.1128/MCB.22.7. 2170-2181.2002)

Chakravarty D, Santos E, Ryder M, Knauf JA, Liao XH, West BL, Bollag G, Kolesnick R, Thin TH, Rosen N et al. 2011 Small-molecule MAPK inhibitors restore radioiodine incorporation in mouse thyroid cancers with conditional BRAF activation. Journal of Clinical Investigation 121 4700-4711. (doi:10.1172/JCI46382)

Dhall A \& Chatterjee C 2011 Chemical approaches to understand the language of histone modifications. ACS Chemical Biology 6 987-999. (doi:10.1021/cb200142c)

Fraga MF, Ballestar E, Villar-Garea A, Boix-Chornet M, Espada J, Schotta G, Bonaldi T, Haydon C, Ropero S, Petrie K et al. 2005 Loss of acetylation at Lys16 and trimethylation at Lys20 of histone $\mathrm{H} 4$ is a common hallmark of human cancer. Nature Genetics 37 391-400. (doi:10.1038/ ng1531)
Horikoshi M 2013 Histone acetylation: from code to web and router via intrinsically disordered regions. Current Pharmaceutical Design 19 5019-5042. (doi:10.2174/1381612811319280002)

Hou P, Liu D, Ji M, Liu Z, Engles JM, Wahl RL \& Xing M 2009 Induction of thyroid gene expression and radioiodine uptake in melanoma cells: novel therapeutic implications. PLoS ONE 4 e6200. (doi:10.1371/ journal.pone.0006200)

Hou P, Bojdani E \& Xing M 2010 Induction of thyroid gene expression and radioiodine uptake in thyroid cancer cells by targeting major signaling pathways. Journal of Clinical Endocrinology and Metabolism 95 820-828. (doi:10.1210/jc.2009-1888)

Kim TH, Park YJ, Lim JA, Ahn HY, Lee EK, Lee YJ, Kim KW, Hahn SK, Youn YK, Kim KH et al. 2012 The association of the BRAF(V600E) mutation with prognostic factors and poor clinical outcome in papillary thyroid cancer: a meta-analysis. Cancer 118 1764-1773. (doi:10.1002/cncr.26500)

Kogai T, Taki K \& Brent GA 2006 Enhancement of sodium/iodide symporter expression in thyroid and breast cancer. Endocrine-Related Cancer 13 797-826. (doi:10.1677/erc.1.01143)

Kuo MH, Brownell JE, Sobel RE, Ranalli TA, Cook RG, Edmondson DG, Roth SY \& Allis CD 1996 Transcription-linked acetylation by Gcn5p of histones H3 and H4 at specific lysines. Nature 383 269-272. (doi:10.1038/383269a0)

Li B, Carey M \& Workman JL 2007 The role of chromatin during transcription. Cell 128 707-719. (doi:10.1016/j.cell.2007.01.015)

Liang G, Lin JC, Wei V, Yoo C, Cheng JC, Nguyen CT, Weisenberger DJ, Egger G, Takai D, Gonzales FA et al. 2004 Distinct localization of histone $\mathrm{H} 3$ acetylation and H3-K4 methylation to the transcription start sites in the human genome. PNAS 101 7357-7362. (doi:10.1073/ pnas.0401866101)

Liu D \& Xing M 2008 Potent inhibition of thyroid cancer cells by the MEK inhibitor PD0325901 and its potentiation by suppression of the PI3K and NF-кB pathways. Thyroid 18 853-864. (doi:10.1089/thy.2007.0357)

Liu Z \& Xing M 2012 Induction of sodium/iodide symporter (NIS) expression and radioiodine uptake in non-thyroid cancer cells. PLOS ONE 7 e31729. (doi:10.1371/journal.pone.0031729)

Liu D, Hu S, Hou P, Jiang D, Condouris S \& Xing M 2007 Suppression of BRAF/MEK/MAP kinase pathway restores expression of iodidemetabolizing genes in thyroid cells expressing the V600E BRAF mutant. Clinical Cancer Research 13 1341-1349. (doi:10.1158/1078-0432. CCR-06-1753)

Momparler RL \& Bovenzi V 2000 DNA methylation and cancer. Journal of Cellular Physiology 183 145-154. (doi:10.1002/(SICI) 1097-4652(200005)183:2<145::AID-JCP1 > 3.0.CO;2-V)

Pugliese M, Fortunati N, Germano A, Asioli S, Marano F, Palestini N, Frairia R, Boccuzzi G \& Catalano MG 2013 Histone deacetylase inhibition affects sodium iodide symporter expression and induces (131)i cytotoxicity in anaplastic thyroid cancer cells. Thyroid 23 838-846. (doi:10.1089/thy.2012.0359)

Puppin C, D'Aurizio F, D'Elia AV, Cesaratto L, Tell G, Russo D, Filetti S, Ferretti E, Tosi E, Mattei T et al. 2005 Effects of histone acetylation on sodium iodide symporter promoter and expression of thyroid-specific transcription factors. Endocrinology 146 3967-3974. (doi:10.1210/en.2005-0128)

Puppin C, Passon N, Lavarone E, Di Loreto C, Frasca F, Vella V, Vigneri R \& Damante G 2011 Levels of histone acetylation in thyroid tumors. Biochemical and Biophysical Research Communications 411 679-683. (doi:10.1016/j.bbrc.2011.06.182)

Puppin C, Passon N, Hershman J, Filetti S, Bulotta S, Celano M, Russo D \& Giuseppe Damante G 2012 Cooperative effects of SAHA and VPA on NIS gene expression and proliferation of thyroid cancer cells. Journal of Molecular Endocrinology 48 217-227. (doi:10.1530/JME-11-0063)

Riesco-Eizaguirre G, Gutiérrez-Martínez P, García-Cabezas MA, Nistal M \& Santisteban P 2006 The oncogene BRAF V600E is associated with a high risk of recurrence and less differentiated papillary thyroid carcinoma due to the impairment of $\mathrm{Na}+/ \mathrm{I}-$ targeting to the membrane. Endocrine-Related Cancer 13 257-269. (doi:10.1677/erc.1.01119) 
Ryu KY, Tong Q \& Jhiang SM 1998 Promoter characterization of the human $\mathrm{Na}+/ \mathrm{I}$ - symporter. Journal of Clinical Endocrinology and Metabolism $\mathbf{8 3}$ 3247-3251. (doi:10.1210/jc.83.9.3247)

Tong Q, Ryu KY \& Jhiang SM 1997 Promoter characterization of the rat $\mathrm{Na}+/ \mathrm{I}-$ symporter gene. Biochemical and Biophysical Research Communications 239 34-41. (doi:10.1006/bbrc.1997.7432)

Vogelauer M, Wu J, Suka N \& Grunstein M 2000 Global histone acetylation and deacetylation in yeast. Nature $\mathbf{4 0 8} 495-498$. (doi:10.1038/ 35044127)

Woo YH \& Li WH 2012 Evolutionary conservation of histone modifications in mammals. Molecular Biology and Evolution 29 1757-1767. (doi:10.1093/molbev/mss022)

Xing M 2005 BRAF mutation in thyroid cancer. Endocrine-Related Cancer 12 245-262. (doi:10.1677/erc.1.0978)

Xing M 2007a BRAF mutation in papillary thyroid cancer: pathogenic role, molecular bases, and clinical implications. Endocrine Reviews $\mathbf{2 8}$ 742-762. (doi:10.1210/er.2007-0007)

Xing M $2007 b$ Gene methylation in thyroid tumorigenesis. Endocrinology 148 948-953. (doi:10.1210/en.2006-0927)
Xing M 2013 Molecular pathogenesis and mechanisms of thyroid cancer. Nature Reviews. Cancer 13 184-199. (doi:10.1038/nrc3431)

Xing M, Westra WH, Tufano RP, Cohen Y, Rosenbaum E, Rhoden KJ, Carson KA, Vasko V, Larin A, Tallini G et al. 2005 BRAF mutation predicts a poorer clinical prognosis for papillary thyroid cancer. Journal of Clinical Endocrinology and Metabolism 90 6373-6379. (doi:10.1210/jc.2005-0987)

Xing M, Haugen BR \& Schlumberger M 2013a Progress in molecular-based management of differentiated thyroid cancer. Lancet 381 1058-1069. (doi:10.1016/S0140-6736(13)60109-9)

Xing M, Alzahrani AS, Carson KA, Viola D, Elisei R, Bendlova B, Yip L, Mian C, Vianello F, Tuttle RM et al. 2013b Association between BRAF V600E mutation and mortality in patients with papillary thyroid cancer. Journal of the American Medical Association 309 1493-1501. (doi:10.1001/jama.2013.3190)

Xu J \& Hershman JM 2006 Histone deacetylase inhibitor depsipeptide represses nicotinamide $N$-methyltransferase and hepatocyte nuclear factor-1 $\beta$ gene expression in human papillary thyroid cancer cells. Thyroid 16 151-160. (doi:10.1089/thy.2006.16.151)

Received in final form 11 November 2013

Accepted 14 November 2013

Made available online as an Accepted Preprint

15 November 2013 http://erc.endocrinology-journals.org

DOI: 10.1530/ERC-13-0399
(C) 2014 The authors Printed in Great Britain
Published by Bioscientifica Ltd. 